\title{
Optimization Based Integrated Fuzzy Logic PID Controller for Networked Controlled Systems (NCSs)
}

\author{
Debashish Pal*, Shiv Kumar Bhagat \\ Department of Electrical, North Eastern Regional Institute of Science and Technology (NERIST), India
}

Received August 12, 2019; Revised December 21, 2019; Accepted December 25, 2019

Copyright $@ 2019$ by authors, all rights reserved. Authors agree that this article remains permanently open access under the terms of the Creative Commons Attribution License 4.0 International License

\begin{abstract}
Nowadays, real-time control networks are becoming an emerging trend in the application of several fields like in remote campaign telecasting. Networked control systems (NCSs) are denoted as spatially distributed systems where the actuators, controllers and sensors are linked over a common band-limited wireless/wired digital communication network. The communication of data in the network plays a pivotal role in a system for its accurate working and as such the network-induced delays becomes a major challenging factor in the transmission of data from one source to another source especially for systems with real-time applications. Therefore, this delay factor should be analyzed and improved further. To overcome this network-induced delay factor along with problems of packet dropouts, fault occurrence and also increasing the stability, a novel technique through this paper is proposed. Here, initially the parameter initialization is done and transmission of data in the network is estimated and evaluated by the use of PID controller which is responsible for estimating the delay factors at the time of communication of data between the nodes. Also, a fuzzy logic parameter is integrated with PID controller to get a measure of probabilistic delay and fault occurrence. Finally, output of this network system is improved by using a novel optimization technique like an improved grey wolf optimization and an enhanced whale optimization. Finally, the performance analysis is done and results thus obtained validates the utility of this method in terms of lower amount of delays with lesser packet dropouts and an enhanced accuracy level in comparison to the traditional methods.
\end{abstract}

Keywords Networked Controlled Systems, PID Controller, Fuzzy Logic, Delays, Grey Wolf Optimization, Whale Optimization

\section{Introduction}

In recent years, due to an increasing trend in real time control networks, networked systems have established wide ranging considerations from investigators in several fields including remote sensing applications. Basically, Networked Controlled System (NCS) is an incorporation of computer science, network technology and control science [1-3]. Its advantages include modest maintenance and installation, reduced costs, condensed weight and power necessities and ease of operation among many others [4]. But due to constraints like constrained bandwidth and restricted service in communication systems, NCSs find themselves with several challenges like delays, packet losses, packet disordering, multipacket transmission etc.

Researches in the fields of NCS have revealed that random delays are the major concern for performance degradation as well as instability. Problems of packet losses, multipacket transmission etc are related directly or indirectly with delays only. The proliferation in energy demand united with the increasing disquiets of global warming [5] has demanded the incorporation of renewable energy support such as wind and solar energy integrated by the power grid. This has given growth to storage systems and hybrid distribution of energy. The energy generation from the solar and wind power plants is generally stochastic and is determined by the climatic conditions at any given time period. This randomness becomes handy wherever the demand is more than the generation. Also, the storage devices when integrated in the process, add to the destabilization already present [6,7]. This whole issue adds to the design challenges in NCS.

In the application of NCS, the sample period is a scheme input variable contingent of the system conditions. Decreasing the sampling time would result in increased delays and system blocking. Hence choosing proper sampling time is a necessary pre-requisite to lessen packet 
dropouts and transmission delays which are a major concern that can lead to destabilization of network.

To overcome the issues of delays in NCS, PID controllers were integrated with the system. It is used to estimate the delay and provide corrective measures to deal with it. But PID controllers themselves were plagued by their inefficiency to deal with non-linear random delays. For these types of delays, researchers were forced to look for other alternatives from which the use of Fuzzy Logic Controllers came into picture. FLC has become more conjoint in modern control applications to hold compound non-linear procedures. It has been revealed by several modern investigators that the use of FLC increases the performance of a PID controller by means of managing modifications in a functional point for non-linear processes in online updating the controller factors. FLCs usually work by regular control rules, resulting from authority's information.

Also, in addition to the problem of tackling delays, increasing the accuracy along with efficiency and learning time of the system has also become one of the paramount challenges for researchers. For these, several optimization techniques are studied and are being integrated with NCS to give better results. Advanced optimization techniques are continuously being studied and developed among which developing methods based on hunting patterns of animals like wolf, whale, bats etc. is attracting particular interests.

In this work, PID controller is integrated with Fuzzy logic to monitor and evaluate the data communication stream in the network. Particularly, PID controller is responsible for monitoring the communication flow, time taken to send data and evaluation of packet dropouts during the transmission process. Fuzzy logic helps to attain the efficiency of the PID controller. In subsequent steps, transfer function of the system is derived and results thus obtained are optimized using Grey Wolf optimization technique and Whale optimization technique which in turn helps in the increase of the performance of the system.

The remaining portion of this paper is schematized as follows: section 2 provides the literature reviews of various existing techniques, section 3 describes the problem formulation, proposed work is described in section 4, section 5 deals with the performance analysis while conclusion is done in section 6 .

\section{Related Work}

This section provides the detailed description of literature review of several approaches employed in the networked control system. In [8], the author suggested a scheme of fuzzy parameter and PID controller for the networked systems by an arbitrary delay of time. In this, the delay of communication and the dropouts of packets together with delay for the networked control system were examined. In order to attain the delay value, approximation technique of available delay value was carried. The delay value was the fuzzy adaptive PID controller which was reliant on the particle swarm and genetic algorithm. Three constrictions of PID were used in Online in an attempt to increase the stability of system over MATLAB co-simulation implementation. It shows the fuzzy logic control structure established on particle swarm optimization (PSO) procedure endorse decreased packet dropouts and transmission delays. The Fuzzy PID controllers were adjusted for time domain optimality with PSO and GA to hold the opposing belongings of stochastic difference of system interruption and package failures.

Author in [9] examined the consequence of network-induced interruption on the dependability of NCSs. At that phase, guiding stochastic structures of the time disruption, an altered Smith interpreter was attached to eliminate the exponential determination of the time delay in the closed-loop characteristic correspondence of the NCS. Similarly, Fuzzy PID algorithm by the fuzzy immune control procedure was united and presented as a fuzzy resistant self-adaptive PID algorithm to recompense the effect of the typical deviance of the measured entity. Finally, a type of fuzzy protected self-adaptive PID procedure depending on new Smith analyst was accessible to relate to the NCS. The outcome demonstrated that the projected control algorithm has the compensations of fast regulating phase and slight overextend, thus it can expand the enactment of the NCS and can attain the anticipated result.

Author in [10] designed an actual and effective insignificant order PID controller from interpretation of a standard PID controller recognized on small order calculus, for a technologically advanced control scheme to attain excellent concerts which were of great academic and applied implications. As the viewpoint of multi-objective optimization, this broadside grants a new fractional order PID(FOPID) controller design technique depending on an enhanced multi-objective optimization procedure for an automatic voltage regulator scheme. The issue of scheming FOPID controller for AVR was initially expressed as a multi-objective optimization issue with three unbiased purposes comprising minimization of integral of absolute error, settling time, and total steady-state error. At that time, an enhanced multi order extremal optimization(MOEO) algorithm was projected to resolve this issue by embracing individual-dependent polynomial mutation (PLM) and repeated optimization mechanism. The extension of MOEO-FOPID to more complex practical control systems was the limitation of this method.

In [11] a strategy of a new fuzzy proportional-integralderivative (PID) controller for automatic generation control of two uneven part consistent thermal schemes was presented. For the first stage teaching-knowledge established optimization procedure was functional to attain the limitations of the projected fuzzy-PID controller. The strategy problem is expressed as an optimization problem 
and TLBO was engaged to enhance the constraints of the fuzzy-PID controller. It is practical that teaching learning based on optimization (TLBO) enhanced fuzzy-PID controller improved its dynamic concert by means of overshoot, undershoot and settling time in rate of recurrence and tie-line power deviancy as related to genetic algorithm (GA), particle swarm (PS), search algorithm (SA), and lozi map- based chaotic algorithm (LCOA) dependent PID controllers.

Authors in [12] suggested a Search group algorithm (SGA) which is one of the present nature-inspired heuristics for resolving altered manufacturing optimization difficulties. Alike other evolutionary procedures, SGA undergoes from the limitations of alteration, which was significantly reliant on the problem. The resolution of this was an adaptive constraint mechanism by means of fuzzy logic, specifically fuzzy SGA (FSGA), for enlightening the resolution advantage of the simple SGA. In FSGA, a fuzzy scheme was joined to vigorously regulate the control constraint rate regarding standardized repetition and standardized error value, which were the responses of the structure. To assess the enactment of FSGA, initially, it was matched alongside those of state-of-the-art processes in excess of the well-known standard purposes. Insignificant supposition implication testing is then practical to create system level. As a final point, so as to establish the latent applicability of FSGA in the arena of control, it is approved to design a robust relative integral derivative execution for the network-dependent control scheme dealing with time delays happened in the communication network.

In [13] a strong problem of stabilization in NCS by sampled-data approach over undefined time-varying selection level through the proportional integral (PI) mechanism policy was investigated. The foremost intention of this broadside was to design a formal feedback sample-data PI controller by time variable response suspension so that the subsequent closed-loop scheme is vigorously constant for all acceptable reservations. Depending on the stability system Lyapunov, a different set of delay-based situations was accomplished by means of direct matrix variations to normalize the stability of system and attain the sampled-data PI control design.

Authors in [14] discussed a sum of all the PID controller that were based on increase of altering methods that have been projected in the last decades; utmost of them are down methods. Conversely, in those circumstances where plants were dependent on constant parametric variations or peripheral conflicts, online gains alteration that was a required choice. This was the case of integrated immersed remotely operated vehicles (ROV) where constraints modify in relation to the device it is built-in with. In repetition, some quantity of time is devoted to adjusting the PID improvements of a ROV. An auto-tune PID-like controller created on Neural Networks (NN) was formulated. The NN shows the role of repeatedly valuing the appropriate set of PID improvements that attains attain strength of the scheme.

In [15], a fuzzy protected PID procedure with a different Smith predictor was presented to efficiently decrease the adverse influence of network-induced interruptions in NCS by means of the v-norm kind to decouple the connection plant. Depending on the plant length after decoupling, the predictor was integrated. At that time, a revised fuzzy resistant response control procedure is utilized to adjust PID controller constraints available. Once there was a typical disparity or factor disconcertion, the set-point pursuing enactment and strength of amount produced can be suggestively enhanced. The offered process does not include the network delay estimation method.

Authors in [16] described a new technique of recompensing the insignificant time variable communication delay in the sliding surface. The slight time deferment in the controller to actuator and sensor to controller station is estimated by means of the approximation technique known as Thiran to plan the sliding surface. A discrete-time mode of sliding control regulation was consequent by the anticipated surface that recompenses insignificant delay of time in controller to actuator and sensor to controller networks for unreliable network control systems. The adequate form for closed loop constancy of the method was consequent with the Lyapunov function. Furthermore, this approach was not extended for both single and multiple dropout conditions of the packets which were the major limitation of this method.

In [17] the authors suggested a hybrid power system that offered several independent energy generation methods such as diesel engine, solar photovoltaic, fuel-cell and wind turbine with other energy storage strategies such as the flywheel and battery. The controller was employed in an isolated place while sending and receiving signals, over an unpredictable communication system with stochastic delay. The controller constraints were modified with the use of strong optimization techniques retaining several alternates of PSO and were related with the resultant optimal results. An archival dependent policy has been utilized for decreasing the amount of utility assessments for strong optimization approaches. The results attained from this robust optimization were capable of handling advanced variant in control expansions and commands without major reduction in the performance of system. This was required from the FO control applications, as the strategy was competent to provide differences in the system constraint which possibly would get affected because of the estimation of FO operators with altered recognition approaches and demand of accurateness. However, this approach may fail to design the strategy for complex non-linear and the interrelated power systems.

In [18] an improved quantum-behaved particle swarm optimization (IQPSO) procedure for optimizing the quantification features and mounting aspects of the fuzzy control of the NCS for which traditional understandings 
and approaches were inappropriate that was presented. In the IQPSO process, shortcomings of quantum-behaved PSO (QPSO) process like its early convergence and resident's variety were discarded by presenting search operatives of activity-based costing (ABC) system into the QPSO system as transformation operatives. Optimization of the fuzzy controller is understood by compelling integral time- weighted absolute error (ITAE) directories in place of a fitness function of IQPSO process.

Authors in [19] described a PID control system especially on time deferring structures like NCS. The altered mechanisms which have now been prepared are shortened here like phase margin and gain margin techniques. The entire pivotal point on time delayed structure was the deferments persuaded by network and the loss of packet.

Authors in [20] presented a control-aware quality of service variation-based co-design technique for NCS. The adapter and its quality of service were dependent on discriminated facilities model and customs information. The investigational outcomes check that such a co-design method, wherever the permanence margin regarding a determined system time deferral was engaged into account by the adapter's quality of Service, which can result in virtuous device concerts even with conservative controllers.

\section{Problem Formulation}

NCSs can be denoted as spatially distributed systems where the actuators, controllers and sensors are linked over a common band limited wireless/ wired digital communication network. The communication of data in the network thus plays a pivotal role. Also, NCS is a network formed with a feedback control mechanism. So, the delays introduced through the network at the time of accessing the control loop as well as packet dropouts become a prime concern in the design of an NCS. Through Fuzzy PID controllers, the problems of stochastically varying time delays in the network are substantially tackled but mostly for defined cases. Hence there arises a need for developing a comprehensive method which deals for both defined as well as undefined cases. This is achieved by using improved optimization techniques such as a Grey Wolf and a Whale optimization technique. Through this, system accuracy as well as its speed, both is enhanced to give a comprehensive method that deals with the shortcomings of the traditional methods efficiently.

\section{Proposed Work}

The main outlines of this work are stated as follows:

1. To estimate the communication of data through the use of PID controller and Fuzzy logic.

2. To obtain the mathematical transfer function of the system.

3. To execute two optimization algorithms viz. improved Grey Wolf optimization algorithm and enhanced Whale optimization algorithm.

4. To reduce the network induced delay thereafter.

The overall proposed system is shown below in Fig. 1. 


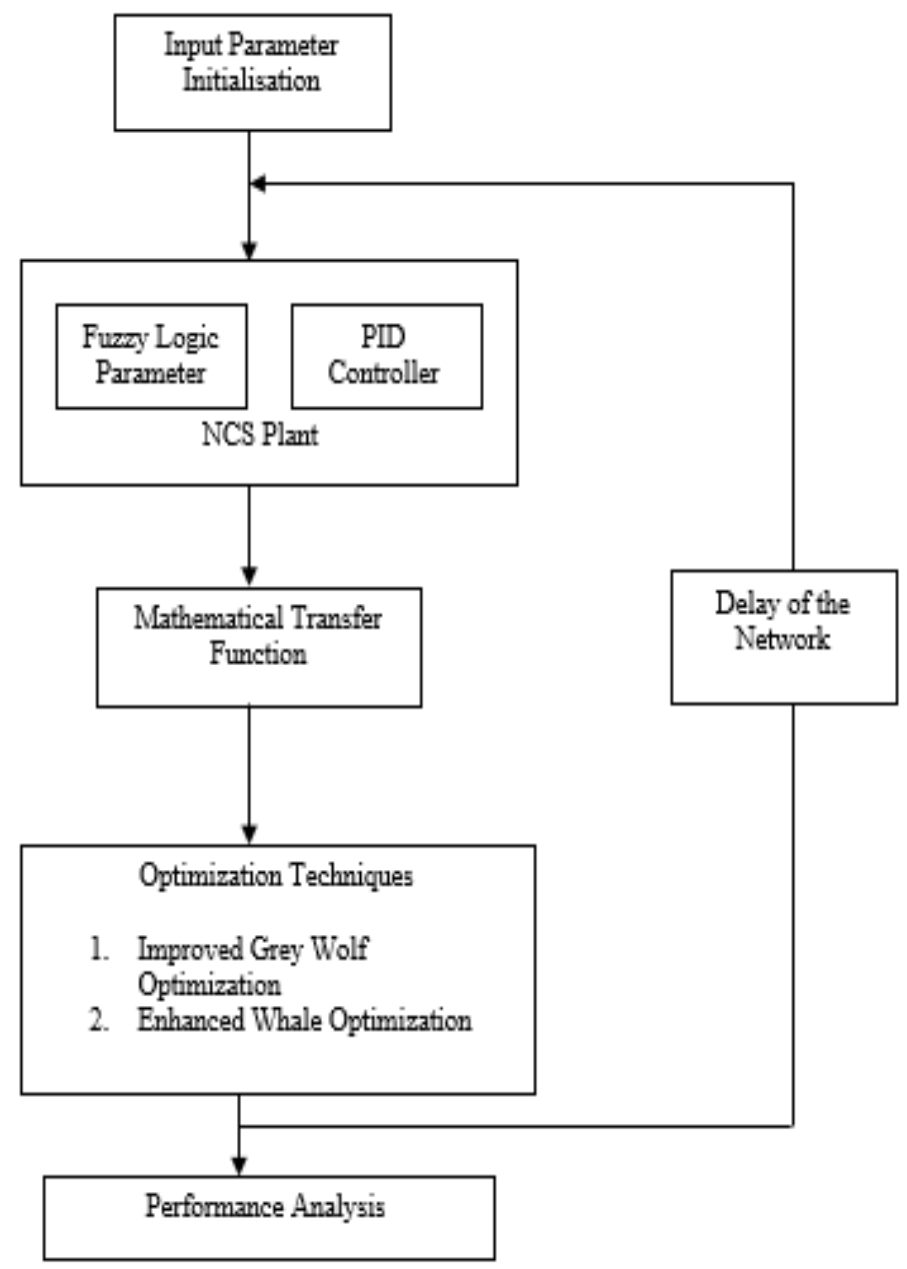

Figure 1. Overall flow of the proposed system

\subsection{NCS Plant}

In this work, initially the inputs are random variables which are fed to the NCS plant after the initialization of parameter is set and done. The plant consists of fuzzy logic parameter and the PID controller. The NCS plant integrates the PID controller with the Fuzzy logic so as to maintain and monitor the stream of data communication of the network system. PID controller is responsible for monitoring the communication flow, time taken to send the data in the network, and faults or packet dropouts during the transmission process. Fuzzy logic is integrated to attain the efficiency of PID controller. There is a problem of broadcast delays, random variation and packet dropouts which can lessen the controller enactment. In NCS, the network delay is the foremost issue producing the classification enactment deprivation. The network delay is adjustable with time, which produces several complications to the control strategy. In current studies, the use of NCS established on fuzzy logic controller is substantiated to be active in production with the difficulties initiated by packet dropouts and transmission delays.

\subsection{Transfer Function}

After the integration of PID and fuzzy logic controller, the mathematical transfer functions are derived. The derived mathematical transfer functions are depicted below:

PID is attuned by the three constraints, grouping of proportional, integral and differential. PID system has decent concert simply in the non-time-varying structure however Fuzzy-PID has nearly adaptive capability to the group delay, time-varying and nonlinear method. A Fuzzy-PID control system has error and its derivative as input factors. Simply, it can be stated that the Fuzzy-PID is united by the benefits of PID and Fuzzy control so as to attain virtuous controller outcome.

The controller output of a conservative PID is a weighted sum of error, its integral and derivative standards.

$$
\mathrm{y}(\mathrm{t})=K_{p} \mathrm{e}(\mathrm{t})+K_{i} \int K_{i} \mathrm{e}(\mathrm{t})+K_{d} d \mathrm{e}(\mathrm{t}) / \mathrm{dt}
$$

Similarly, the Fuzzy PID control parameters can be written as

$$
y_{f u z z y}(\mathrm{t})=K_{p} \mathrm{e}(\mathrm{t})+K_{i} \int K_{i} \mathrm{e}(\mathrm{t})+K_{d} d \mathrm{e}(\mathrm{t}) / \mathrm{dt}
$$


The weighted sum of the control signal on taking the random step size of the input( $\operatorname{ran}_{\text {in }}$ ) can be represented as

$$
\mathrm{w}_{c}=\int\left[w_{i 1} \mathrm{t}(\mathrm{e}(\mathrm{t}))+w_{i 2} \mathrm{t}(\mathrm{e}(\mathrm{t}))\right] \mathrm{dt}
$$

The actuator model, network delay, time delay, transport delay are estimated and are represented as follows:

$$
\begin{gathered}
\text { Actuator Model, } \mathrm{T}=1 /(7 s+1)^{2} \\
\text { Network delay, } \mathrm{T}=0.0003 /\left(8 s^{2}+s\right) \\
\text { Transport delay, } \mathrm{T}=0.001 /(8+s) \\
\text { Time delay, } \mathrm{T}=0.0004 /\left(10 s^{2}+s\right)
\end{gathered}
$$

Fuzzy PID controllers were analyzed on considering the groupings of hybrid controllers by combining the proportional, integral and derivative activities by means of fuzzy interference.

\subsection{Optimization}

The output of the mathematical transfer functions is optimized by an enhanced Grey wolf optimization and whale optimization approach. This technique of optimization is capable of increasing the performance of the network system. It is again optimized by allowing the optimized output to nurture to attain the response. The algorithms for the whale optimization and gray wolf optimization techniques are depicted below:

\section{Algorithm 1 - An enhanced Whale Optimization:}

Initialize the whale's populations $W x_{c}$ (ii= 1, 2, 3...n)

Calculate the fitness of each search agent

$W x_{c}$ is the best search agent

$L s_{C}=\infty$;

$\mathrm{t}=0$;

$i t r_{\text {max }}=$ max no of the iteration

While $\left(t_{w}<i t r_{\max }\right)$

Each agent

Let update the position $\alpha, \theta, \mu, \beta$ and $\rho$

For $\mathrm{i}=1$ : size (update position, 1)Let find objective function for each search agent,

If fitness $<L s_{C}$

$L s_{C}=$ fitness update the alpha $\alpha$

$L s_{p}=$ update position (I, :)

End

End

End

To find the linearity,

$\mathrm{A} 1=2-\mathrm{t}^{*}\left(2 / i t r_{\max }\right)$;

$\mathrm{A} 2=1+\mathrm{t} *\left(\left(-1 /\right.\right.$ itr $\left.\left._{\text {max }}\right)\right)$

Let update the search agent,

For ii=1: size (position, 1)

r1=rand ();

r2=rand ();

Parameters equation,

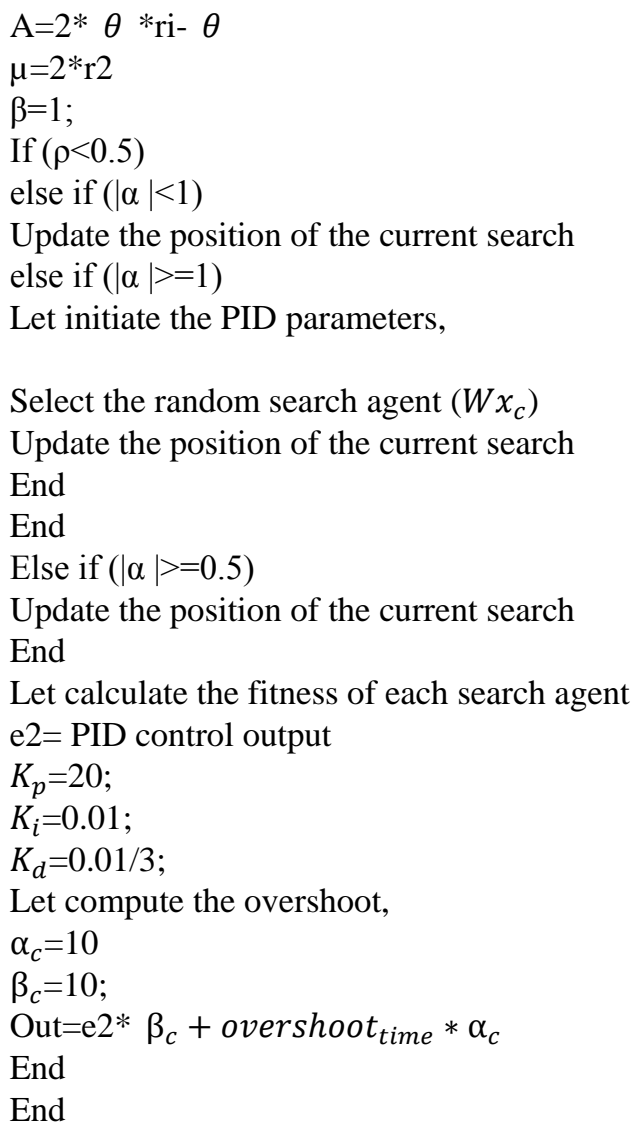

An enhanced whale optimization algorithm is a novel optimization technique. In this, the whale populations are initialized. The fitness function of each search agent is then computed from which the best search agent is found on repeating the number of iterations. After that, the position is being updated as per the size. The objective function of each search agent is identified as per the up dation of fitness function values. Once, the linearity is updated, the position of the present search will be then simplified thereby initiating the PID parameters. From the output of PID control, the overshoot values are computed finally.

\section{Algorithm 2An improved Grey wolf optimizer}

Let initialize the $\alpha, \beta, \theta$ positions

$$
\begin{aligned}
& \alpha_{p}=\text { zeros }\left(1, \mathrm{~d}_{i}\right) \\
& \alpha_{\text {score }}=\infty \\
& \beta_{p}=\text { zeros }\left(1, \mathrm{~d}_{i}\right) \\
& \beta_{\text {score }}=\infty \\
& \theta_{p}=\text { zeros }\left(1, \mathrm{~d}_{i}\right) \\
& \theta_{\text {score }}=\infty \\
& \mathrm{t}=0 ; \\
& i t r_{\text {max }}=\text { max no of the iteration }
\end{aligned}
$$

Let initialize the positions search agents, $\mathrm{l}_{c}=0$;loop counter

While $\mathrm{l}_{c}<i t r_{\max }$ 
For $\mathrm{i}=1$ : size (position, 1 )

Let update $\alpha, \beta, \theta$ positions

If fit $_{\text {val }}<\alpha_{\text {score }}$

$\alpha_{\text {score }}=$ fit $_{\text {val }}$

$\alpha_{\text {pos }}=$ positions (i, :)

End

If fit $_{\text {val }}<\alpha_{\text {score }} \& \&$ fit $_{\text {val }}>\beta_{\text {score }}$

$\beta_{\text {score }}=f i t_{\text {val }}$

$\beta_{p}=$ positions (i, :)

End $<$

If $f i t_{\text {val }}<\alpha_{\text {score }} \& \& f_{i t_{\text {val }}}>\beta_{\text {score }} \& \&$ fit $_{\text {val }}<$

$\theta_{\text {score }}=$ fit $_{\text {val }}$

$\theta_{p}=$ positions (i, :)

End

$\mathrm{A} 1=2-\mathrm{t} *\left(2 / i t r_{\max }\right)$;

$\mathrm{A} 2=1+\mathrm{t}^{*}\left(\left(-1 /\right.\right.$ itr $\left.\left._{\max }\right)\right)$

Let update the search agent,

For ii=1: size (position, 1)

$\tau 1=$ rand () ;

$\tau 2=$ rand ()$;$

Parameters equation,

$\varphi=2 * \theta * \tau 1-\theta$

$\mu=2 *$ r2

$\beta=1$;

If $(\rho<0.5)$
Else if $(|\alpha|<1)$

Update the position of the current search

Else if $(|\alpha|>=1)$

Let calculate the fitness of each search agent

$\mathrm{e} 2=\mathrm{PID}$ control output

$K_{p}=20$

$K_{i}=0.01$;

$K_{d}=0.01 / 3$;

Let compute the overshoot,

$\alpha_{c}=10$

$\beta_{c}=10$

Out $=\mathrm{e} 2 * \beta_{c}+$ overshoot $_{\text {time }} * \alpha_{c}$

End

End

An improved grey wolf optimization algorithm is represented in which the positions are initialized by repeating the number of iterations.

The fitness function values are estimated after the up-dation of position. Finally, the overshoot is computed. The complete architecture of the proposed technique is shown below in Fig. 2. 


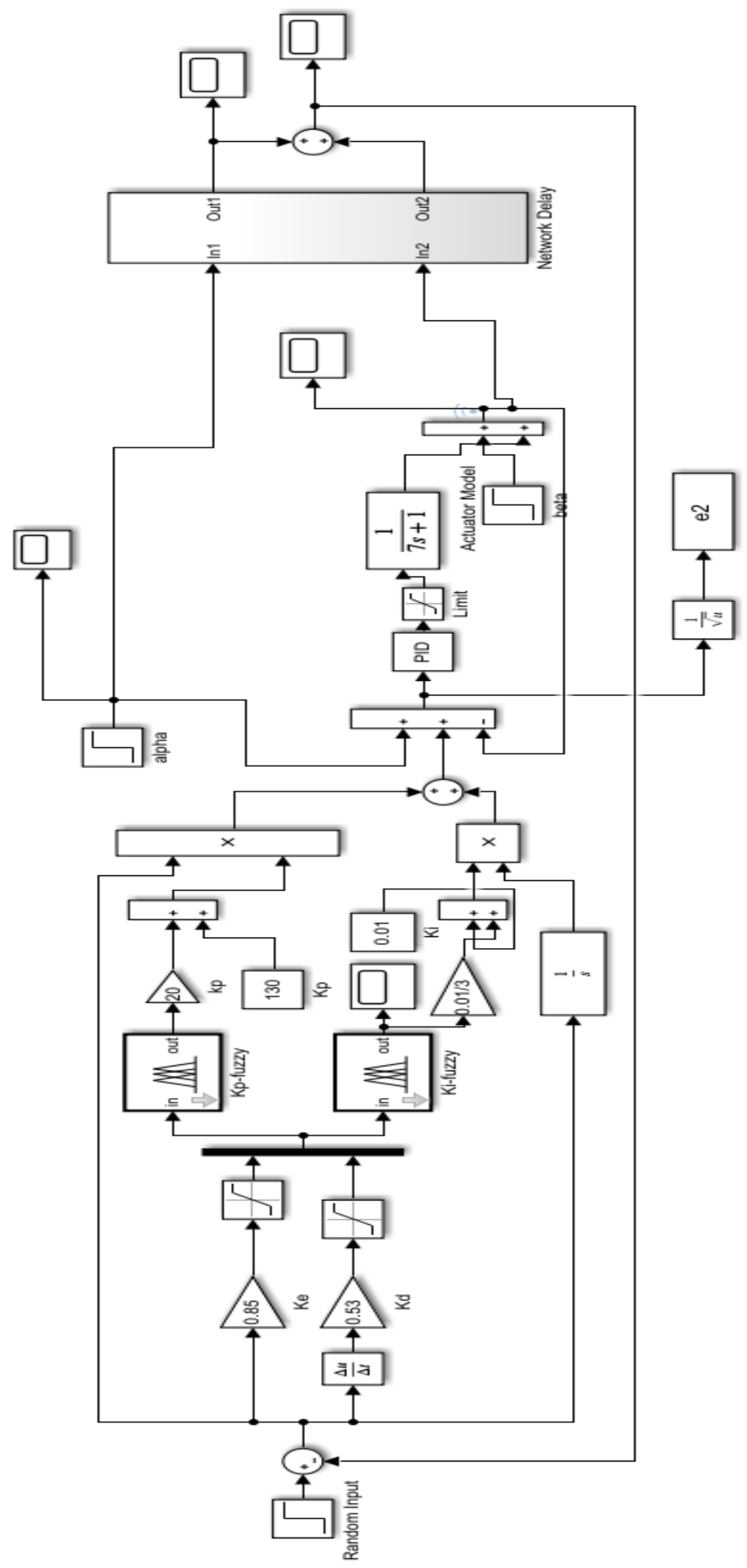

Figure. 2. System architecture 


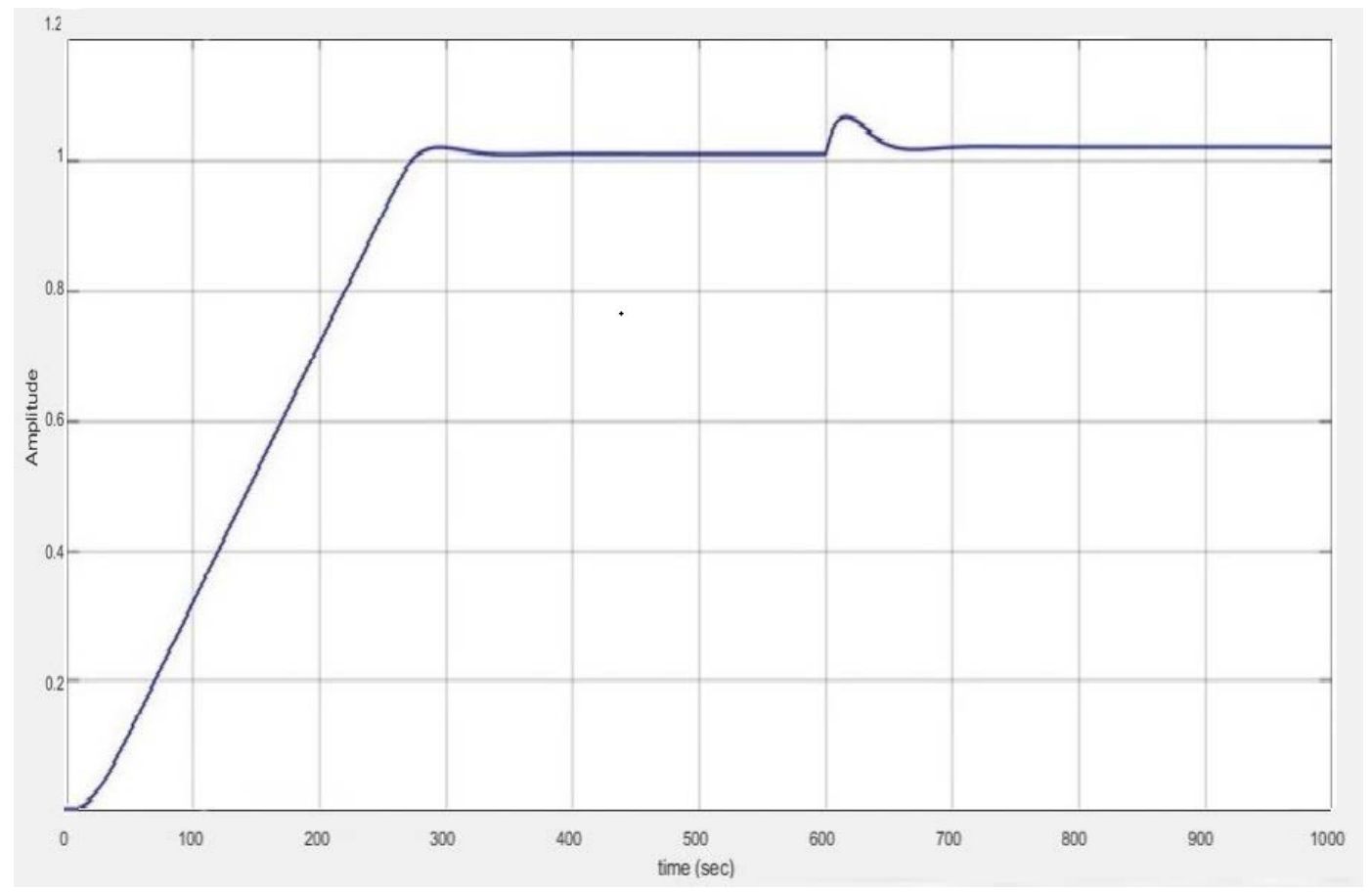

Figure. 3. Delay without optimization technique

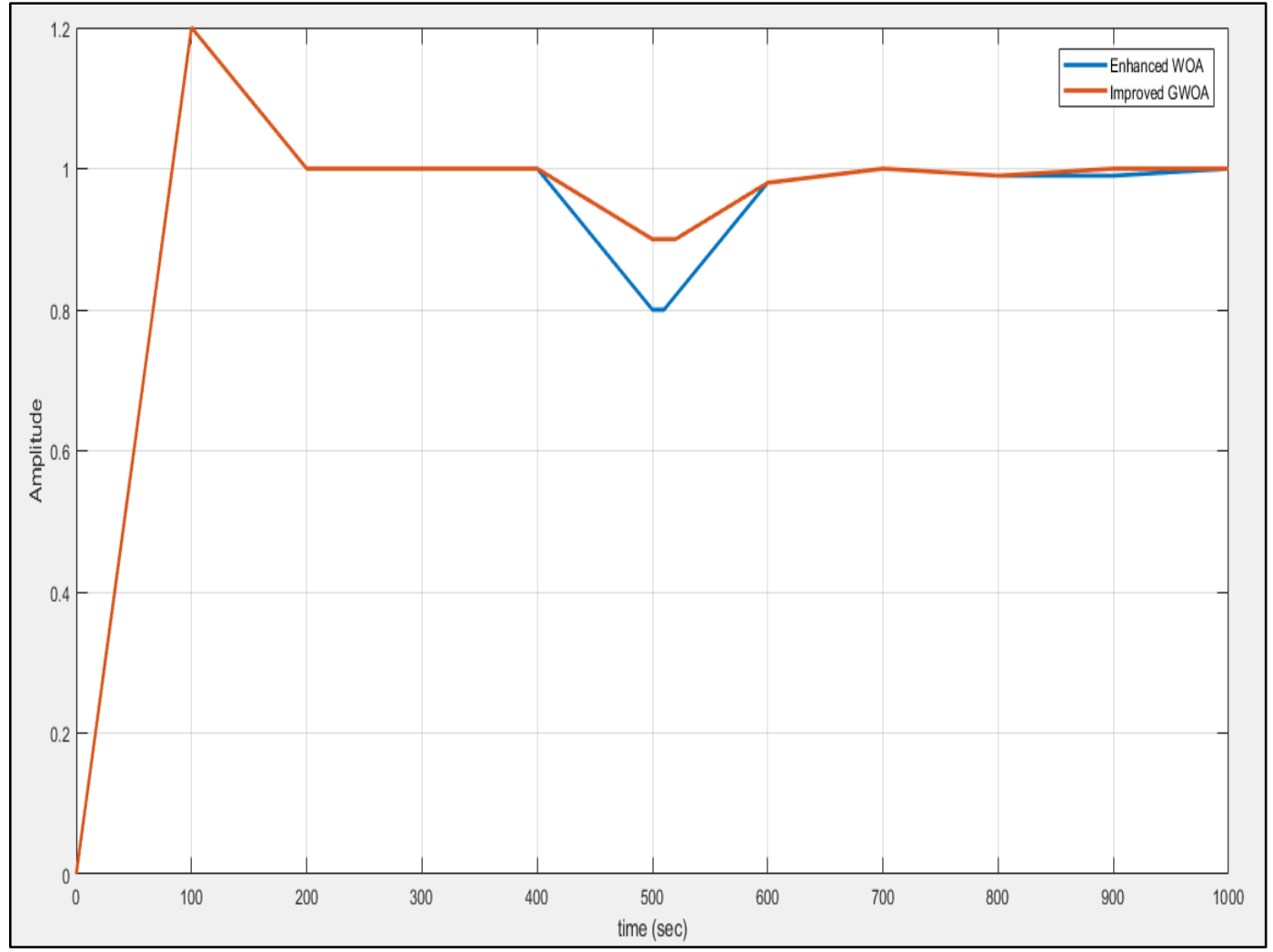

Figure. 4. Delay with optimization technique 


\section{Performance Analysis}

The proposed work is implemented in MATLAB and results are obtained using Fuzzy PID controller with the application of optimization algorithms.

Fig. 3 shows the delay estimation in the existing methods while Fig. 4 the delay estimation for the proposed method.

The comparative analysis of the existing and proposed technique is shown in terms of delay estimation in the network in Fig. 5.

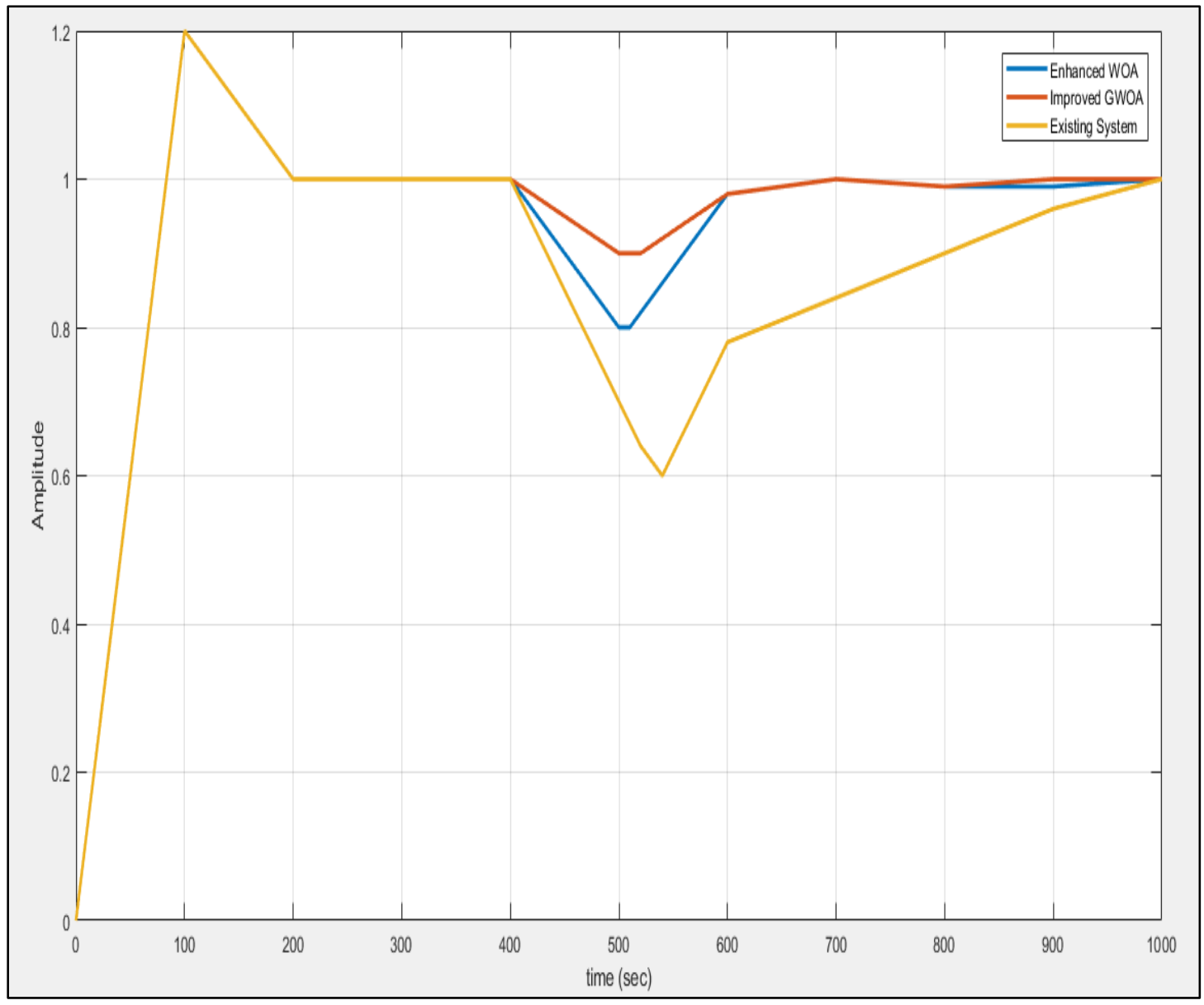

Figure. 5. Comparison of Delays 
From the above analysis it is evident that our proposed technique with the use of optimization techniques like improved grey wolf and enhanced whale optimization offers a lower rate of delay on comparing the delay estimation made without the use of optimization techniques. Therefore, the proposed technique is much more efficient than the other existing techniques.

\section{Conclusions}

The major issues encountered in an NCS like stability, packet loss, more computing time etc could be attributed primarily to the delays introduced in the network. Hence tackling the issue of delays becomes one of the major design challenges for an NCS owing to its widespread use. Therefore, in this regard, the use of optimization technique like Grey Wolf and Whale optimization has helped in the reduction of delays which are validated from the results obtained. Thereby the proposed method helps in getting a better NCS design in terms of higher efficiency, lower packet loss, higher stability and less computing time.

\section{REFERENCES}

[1] Y.L Wang., et al. Event-triggered fault detection filter design for a continuous-time networked control system, IEEE transactions on cybernetics, vol. 46, 3414-3426, 2016.

[2] Y. Luo., et al. Fuzzy-logic-based control, filtering, and fault detection for networked systems: A Survey, Mathematical problems in Engineering, Hindawi publishing corporation, 2015.

[3] R. Lu., et al. A new design of model predictive tracking control for networked control system under random packet loss and uncertainties, IEEE Transactions on Industrial Electronics, vol. 63, 6999-7007, 2016.

[4] X.M.Zhang., et al. An overview and deep investigation on sampled-data-based event-triggered control and filtering for networked systems, IEEE Transactions on Industrial Informatics, vol. 13, 4-16, 2017.

[5] I.Pan., et al. Fractional order fuzzy control of hybrid power system with renewable generation using chaotic PSO, ISA transactions,vol. 62, 19-29, 2016.

[6] G.C.Sekhar., et al. Load frequency control of power system under deregulated environment using optimal firefly algorithm, International Journal of Electrical Power \& Energy Systems,vol. 74, 195-211, 2016.

[7] D.Zhang., et al. Network-Based PI Control for Output Tracking of Continuous-Time Systems With Time-Varying Sampling and Network-Induced Delays, Journal of the Franklin Institute, 2018.

[8] F.Liu., et al. The Design of Fuzzy PID Controller for Networked Systems with Random Time Delay, International Journal of Grid and Distributed
Computing,vol. 9, 117-124, 2016.

[9] H.Zhang., et al. Research on fuzzy immune self-adaptive PID algorithm based on new smith predictor for networked control system, Mathematical Problems in Engineering, 2015.

[10] G.Q. Zeng., et al. Design of fractional order PID controller for automatic regulator voltage system based on multi-objective extremal optimization, Neurocomputing, vol. 160, 173-184, 2015.

[11] B.K. Sahu., et al. Teaching-learning based optimization algorithm based fuzzy-PID controller for automatic generation control of multi-area power system, Applied Soft Computing, vol. 27, 240-249, 2015.

[12] S. F. H. Noorbin., et al. Adaptive parameter control of search group algorithm using fuzzy logic applied to networked control systems, Soft Computing, vol. 22, 7939-7960, 2018.

[13] S. Santra., et al. Robust sampled-data PI controller design for networked control systems, Journal of the Franklin Institute, vol. 353, 797-815, 2016.

[14] R. H. Alvarado., et al. Neural network-based self-tuning PID control for underwater vehicles, Sensors,vol. 16, 1429, 2016.

[15] Y. Tang., et al. New Smith predictive fuzzy immune PID control algorithm for MIMO networked control systems, EURASIP Journal on Wireless Communications and Networking, 212, 2018.

[16] D.Shah., et al. Fractional delay compensated discrete-time SMC for networked control system, Digital Communications and Networks, vol. 3, 112-117, 2017.

[17] I. Pan., et al. Fractional order AGC for distributed energy resources using robust optimization, IEEE Transactions on Smart Grid, vol. 7, 2175-2186, 2016.

[18] W. Zoujun. Fuzzy Self-Tuning PID Control Method Based on Multi-Agent Particle Swarm Algorithm, International Journal Of Simulation--Systems, Science \& Technology, vol. 17, 1-6, 2016

[19] D.S. Sahu and S. Sharma. A survey paper on PID control system, International Journal of Engineering Trends and Technology (IJETT), vol. 21, 366-368, 2015.

[20] O. Stefan., et al. On the Robustness of Networked Control Systems with Quality of Service Adaptation Co-design, Journal of Control Engineering and Applied Informatics, vol. 18, 57-64, 2016. 